\title{
Theoretical and Experimental Study on the Water Curtain Shape and Mechanical Relationship of the Smoke Control System
}

\author{
GONG Hongwei ${ }^{1,2,3, a^{*}}$, JIANG Juncheng ${ }^{1,2,3}$ QIU Jinming ${ }^{1}$, JIANG Ranran¹, \\ ZHANG Weikang ${ }^{1}$
}

${ }^{1}$ Nanjing Tech University, Nanjing 21000, China

2 Jiangsu Key Laboratory of Hazardous Chemicals Safety and Control, Nanjing 210000, China,

${ }^{3}$ Nanjing University of Technology, Institute of Fire Science and Engineering, Nanjing 210000,

China

asanwen@njtech.edu.cn

\begin{abstract}
Keywords: Smoke control system, Continuous water curtain, Mechanics mechanism, Water surface tension, Net suction.
\end{abstract}

\begin{abstract}
First, we conduct the research on the mechanical mechanism of smoke control system to form a continuous water curtain and analysis the mechanical model of continuous water curtain and micro-unit; then simulate the continuous water curtain's shape of smoke prevention system by using Fluent software and the results are compared with the theoretical analysis; Finally, adopting the experimental verification of continuous water curtain shape and analyzing shape change of continuous water curtain in different water surface tension. Studies have shown that:1) the water curtain boundary track is curved, but the water curtain was similar to the shape of inverted trapezoid.2) under conditions of lowering the surface tension of water (test conditions), and continuous water curtain area growth rate with an average value is $0.26 \%$, the width of growth rate with an average value is $17.32 \%$, the length of the growth rate with an average value is $-5.19 \%$; Results indicates that the decrease of surface tension will cause the width of water curtain increase, the length is reduced, but it has less effect to the area of water curtain.
\end{abstract}

\section{Introduction}

The mainly hazards in building fire are functioned by three factors: heat radiation, smoke and hypoxia, but for most of the fires, smoke caused the largest proportion of casualties relative to the damage caused by heat radiation and hypoxia. The traditional way of smoke control mainly include natural smoke exhaust and mechanical smoke exhaust [1]; setting smoke separation for buildings, fire shutter, smoke hanging wall and the air curtain, etc. These traditional ways of smoke control have some certain effect on fire prevention and smoke insulation, but there are some defects though, such as front room of staircase for natural smoke exhaust must be designed rely on external wall, or it is difficult to achieve the effect, and is subject to the influence of natural wind, which may cause an unstable result; In the case of mechanical pressurization anti smoke, because there are many factors that affect the stress calculation of air quantity, the pressurized total air volume of anti-smoke staircase and vestibule, combined compartment and fire elevator foyer need to be determined by calculation, the design calculation is complex; Air curtain as the mean of smoke preventing, it has the inconvenient problem of large volume, inconvenient installation, and high installation costs, etc.

Water curtain is a new way of smoke control, it is the only automatic sprinkler system which does not set putting out fire as the main purpose, the system is used in the building smoke control system, not only has the effect of putting out the fire and preventing smoke, but also a kind of energy saving and environmental protection model of green fire control system. But traditional water curtain system is simply connect a water supply pipe with the nozzle, spraying into water curtain shape by high pressure, flue gas can go through the gap in the middle of the water curtain at the time of fire. Therefore it cannot completely stop the smoke from spreading, and traditional 
water curtain system needs a large quantity of water and has poor resistance effect of preventing smoke.

Based on the disadvantages of traditional smoke prevention system and traditional water curtain system, putting forward a kind of "new preventing smoke water curtain system", the system consists of water supply source, piping system, water tank, recovery system and automatic control system. The key to the system is that the water curtain system will open and form a layer of uniform and continuous overflow water curtain to prevent the spread of smoke and heat radiation after the fire happen, and the formation of continuous water curtain is influenced by many factors. In this paper, theoretical analysis, numerical simulation and experiment [2] will be conducted on how boundary force will affect continuous water curtain.

\section{Mechanics Mechanism of Forming a Continuous Water Curtain}

Mechanical Model of Continuous Water Curtain. After the water outflows(average amount) from the tank of water curtain system, the continuous water curtain will shape into a layer of smooth overflow due to mutual forces $U$, which actually is the intermolecular force that acting between molecules, also known as the van der Waals forces, form the connection between water molecules. On the interface between water and air, since the pressure on the surface of the water molecules is not even, an inward contraction force will be generated on the water surface, which is the net suction $F$ (Intermolecular forces can cause net suction, and the net suction can cause surface tension, surface tension is tangent with liquid surface forever, and is perpendicular with net suction). Continuous water curtain is not only affected by the internal force of van der Waals force, but also affected by external force, including atmospheric pressure $P$, the air resistance $\mathrm{f}$ and gravity $G$, the mechanical model is shown in Fig. 1(set $0 y$ (set 0 for coordinate $(0,0)$ )as the line of symmetry).

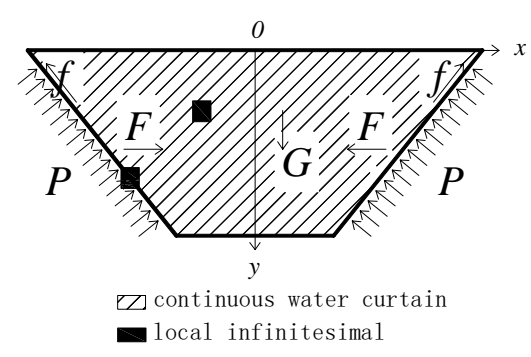

Fig. 1 Mechanical model of continuous water curtain.

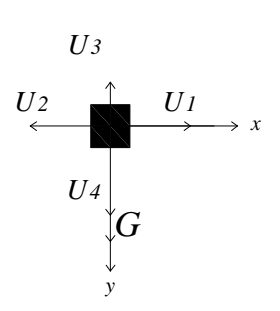

Fig. 2 Internal micro-unit force model.

Internal Micro-unit Force Analysis of Continuous Water Curtain. Force analysis is performed on the internal micro-unit in mechanical model (Fig. 1). The main force acting on the xoy surface includes van der Waals force $U$ and gravity $G$. There are several forces on the micro-unit: van der Waals forces $U_{1}$ along the $\mathrm{x}$ axis direction, van der Waals force $U_{2}$ along the -x axis, gravity $G$, and van der Waals force $U_{4}$ along the $\mathrm{Y}$ axis direction, and van der Waals force $U_{3}$ along the $-\mathrm{y}$ axis direction. The mechanics model is shown in Fig. 2. Van Edward force size order: $U_{3}<U_{2}=U_{1}<$ $U_{4}$.

Analysis formula of the internal micro-unit:

Resultant force of the $\mathrm{x}$ axis, forward direction along the $\mathrm{x}$ axis: $F_{x}=U_{1^{-}} U_{2}=0$;

Resultant force of the y axis, forward direction along the y axis: $F_{y}=G+U_{4}-U_{3}>G>0$.

Results of force analysis of continuous water curtain internal micro-unit: 1) Along the $\mathrm{x}$ axis direction, the resultant force $\mathrm{Fx}=0$, therefore, there is no displacement on the $\mathrm{x}$ axis direction; 2 ) Along the $\mathrm{y}$ axis direction, the resultant force Fy $>0$, there is acceleration motion on the $\mathrm{y}$ axis direction.

Boundary Micro-unit Force Analysis of Continuous Water Curtain. Force analysis is performed on the boundary micro-unit.. The main force acting on the xoy surface includes net suction F, atmospheric pressure $\mathrm{P}$, pressure between liquid $\mathrm{Pr}$, air resistance $\mathrm{f}$ and gravity $\mathrm{G}$. Since the boundary of continuous water curtain is affected by net suction, atmosphere pressure and some 
other factors, so there is an angle $\alpha(0<\alpha \leq \pi / 2)$ between the boundary and the y axis, the micro-unit is affected by gravity $\mathrm{G}$ along y direction, air resistance $\mathrm{f}$ (upward direction along the interface) along -y direction angle $\alpha$, atmospheric pressure $\mathrm{P}$ (upward direction along the interface) along the $\mathrm{x}$ axis angle $\alpha$, net suction $\mathrm{F}$ along the $\mathrm{x}$ axis angle $\alpha$. The mechanical model is shown in Fig. 3 . $P=P r$.

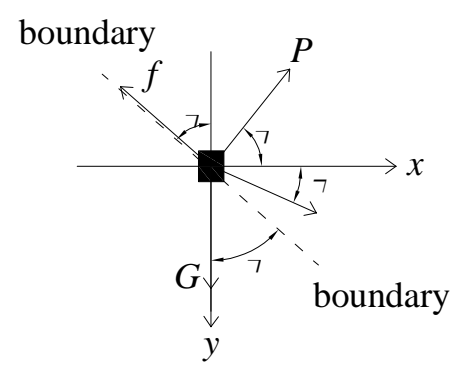

Fig. 3 Boundary surface micro-unit force model.

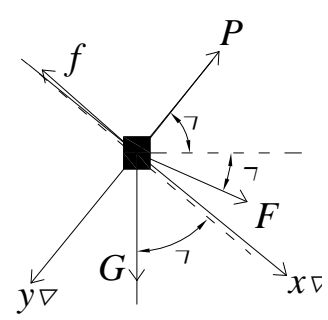

Fig. 4 Boundary surface micro-unit force model ( $x^{\prime} y^{\prime}$ axis)

Analysis formula of the micro-unit on boundary:

Resultant force of the $\mathrm{x}$ axis, forward direction along the $\mathrm{x}$ axis: $F_{x}=F \cos \alpha+P \cos \alpha-f \sin \alpha-$ $P r \cos \alpha=F \cos \alpha-f \sin \alpha$;

Resultant force of the $y$ axis, forward direction along the $y$ axis: $F_{y}=G+$ $P r \sin \alpha-F \sin \alpha-P \sin \alpha-f \cos \alpha=G-F \sin \alpha-f \cos \alpha$.

While the water curtain falling, air resistance f gradually increased from 0 , angle $\alpha$ also changes, atmospheric pressure $\mathrm{P}$ remains the same. In order to research boundary flow path more conveniently, the tangent direction of boundary is refered as $\mathrm{x}$ axis, vertical direction is refered as $\mathrm{y}^{\prime}$ axis. The mechanical model is shown in Fig. 4.

Analysis formula of the micro-unit on boundary:

Resultant force of the $\mathrm{x}$ axis, forward direction along the $\mathrm{x}$ axis: $F_{x^{\prime}}=G \cos \alpha-f$;

Resultant force of the y axis, forward direction along the y axis: $F y^{\prime}=P r-P+G \sin \alpha-F=G \sin \alpha-F$

\section{Analysis of Continuous Water Curtain Shape.}

Analysis of boundary flow path. As fluid flows out of the tank port $(y=0)$, due to the surface tension of the water (essentially is net suction), the water shrink inward, if the water amount is insufficient, resultant force on water boundary surface Fy $<0$, the angle $\alpha=\pi / 2$, at this point the water will flow inside along the outlet wall. With the increase of flow amount until gravity reaches a certain critical value $\mathrm{G}_{0}$, gravity surface tension, $\alpha$ gradually decreases ( $\downarrow$ represents reduction in formula), F remains the same, f gradually increases $(\uparrow$ represents increase in formula),

Analysis of resultant force change on the $\mathrm{x}$ axis and y axis of boundary surface: $F_{x}=F \cos \alpha \uparrow-$ $f \uparrow \sin \alpha \downarrow ; F_{y}=G-F \sin \alpha \downarrow-f \uparrow \cos \alpha \uparrow$. The index values in formula $F_{x}$ and $F_{y}$ increases and reduces, resultant force change on the $\mathrm{x}$-axis and $\mathrm{y}$-axis cannot be judged.

Analysis of resultant force change on the $x^{\prime}$ axis and $y^{\prime}$ axis of boundary surface: $F_{x^{\prime}}=G \cos \alpha \uparrow$ $f \uparrow ; F_{y^{\prime}}=G \sin \alpha \downarrow-F$. The index values in formula $F_{x^{\prime}}$ all increases, resultant force change on the $\mathrm{x}$-axis and y-axis is not proper to be judged. $G \sin \alpha$ in formula $F_{y^{\prime}}$ decreases, and $F_{y^{\prime}} \geqslant 0$, therefore, with the change of angle $\alpha$, resultant force on y 'axis tends to zero, and $\alpha$ tends to certain value.

Provided $F y^{\prime}=G \sin \alpha-F=0$, the $\sin \alpha=F / G$, so angle $\alpha$ is proportional to the net force $\mathrm{F}$.

Through the above theoretical analysis: angle between boundary flow path and y axis first decreases slowly, then rapidly forming an angle $\alpha$, then slowly decreases, so the boundary flow path shaped in curve, the continuous water curtain shape is shown in Fig. 5. 


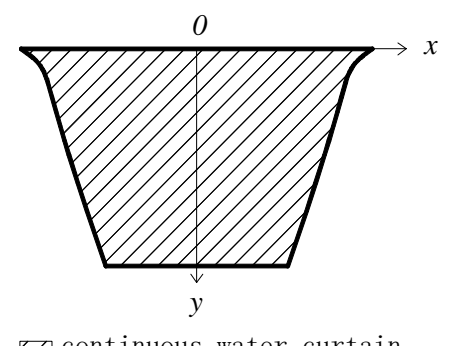

$\square$ continuous water curtain

Fig. 5 Continuous water curtain shape.

Velocity analysis of the continuous water curtain. When doing the force analysis of micro-unit, the resultant force of internal micro-unit on y axis is $F_{y}=G+U_{4}-U_{3}$, the resultant force of boundary micro-unit on y axis is $F_{y}=G-F \sin \alpha-f \cos \alpha$. Since $U_{4}-U_{3}>0$, so the resultant force of internal micro-unit on y axis is $F_{y}>G$, and the resultant force of boundary micro-unit on y axis is $F_{y}<G$. Therefore the resultant force of internal micro-unit on y axis is bigger than the resultant force of boundary micro-unit on y axis, which means the internal micro-unit has bigger downward acceleration than boundary micro-unit. As a result, in the condition of the same continuous water curtain length, the velocity on boundary surface is lower than that on the non-boundary surface.

\section{Simulation Studies}

Model Building. In order to verify the conclusions from theoretical analysis, using Fluent software to simulate the shape and the distribution of velocity of water curtain. The model size of the water tank is $200 \mathrm{~mm} \times 80 \mathrm{~mm} \times 130 \mathrm{~mm}$ (length $\times$ width $\times$ height), and the whole calculation domain is $600 \mathrm{~mm} \times 166 \mathrm{~mm} \times 800 \mathrm{~mm}$ (length $\times$ width $\times$ height). Boundary condition is to set the mass-flow-inlet of $0.0734 \mathrm{~kg} / \mathrm{s}$ and the outlet boundary is defined as pressure-out, choose the VOF model, using the pressure-based solver, the water temperature is $20^{\circ} \mathrm{C}$, and the surface roughness constant is 0.5 , the water surface tension is $0.07 \mathrm{~N} / \mathrm{m}$ [5-7].

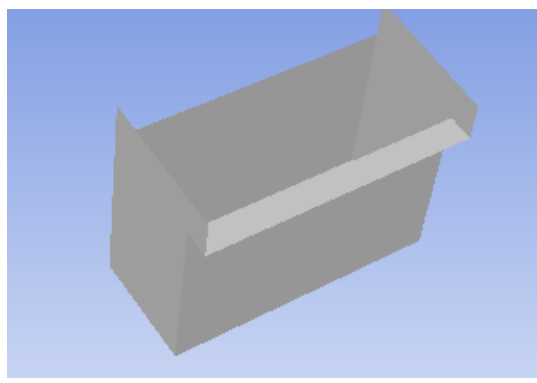

Fig. 6 Tank model.

\section{Simulation Results and Analysis.}

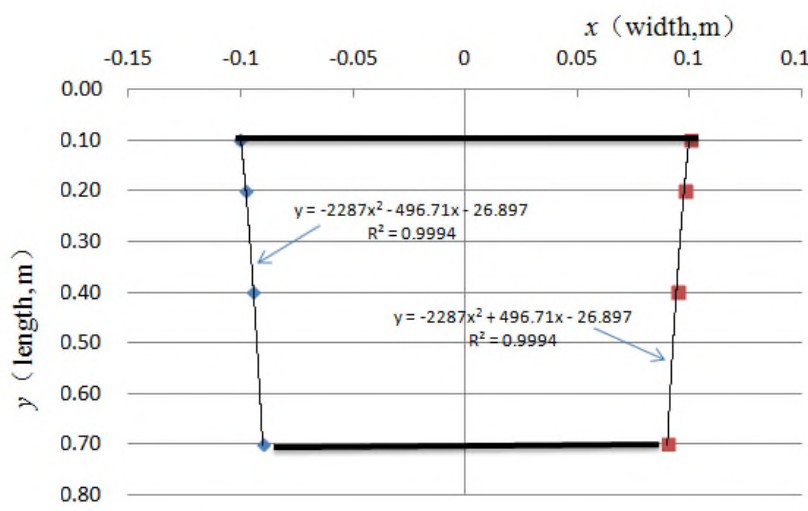

Fig. 8 Continuous water curtain shape.

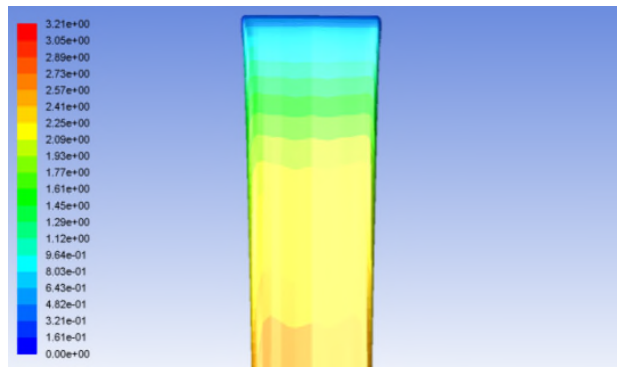

Fig. 7 Velocity contours simulation results.

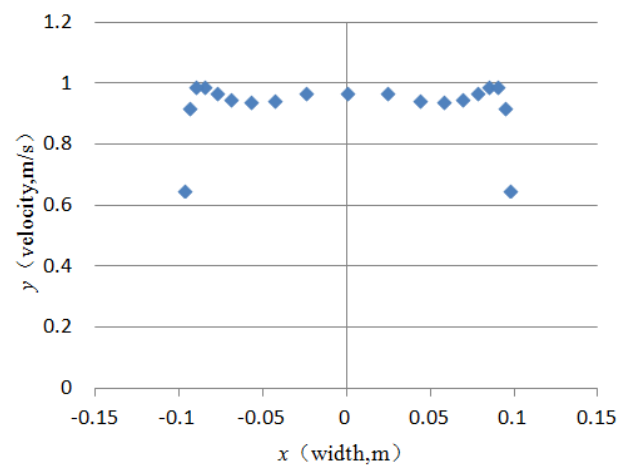

Fig. 9 Sectional velocity distribution based on overflow length $100 \mathrm{~mm}$. 
Simulation results are shown in velocity contours (Fig. 7), through the velocity contours analysis, water curtain boundary appears an overall contraction phenomenon, with the fluid flow rate is keeping increase. In order to get the specific boundary flow path, reading and processing the data from simulation to analyze overflow width under different overflow length. Setting the symmetry plane of water curtain as center and setting center location to zero to obtain the relationship diagram between the length and width of the overflow (Fig. 8). Trending fitting the relationship diagram to get the water curtain boundary flow path. The water curtain shape is in consistent with the conclusion of theoretical analysis.

In order to analyze the relationship between velocities of different points on cross-section of the overflow at the same length, reading the data of the velocity contours of the overflow at the length of $100 \mathrm{~mm}$ (Fig. 9). From the cross-section velocity distribution, we can see that the velocity on water curtain boundary is faster than that on non-boundary. The velocity distribution of the water curtain increases and then slightly decreases, at last increases again along the direction from edge to center, but fluctuation is small. The boundary velocity analysis is consistent with the theoretical analysis, the velocity on water curtain boundary is faster than that on non-boundary.

\section{Experiment Research}

Experiment Subject. The experiment devices mainly includes homemade water tank (The size of the water tank is $1600 \mathrm{~mm} * 210 \mathrm{~mm} * 160$, mm Fig. 10), submersible pump, reservoir, water pipe, steel ruler, detergent etc. The working principle of the system (Fig. 11) is: connecting water pipe to reservoir, submersible pump and water tank, water in the reservoir will be absorbed into the water tank when pump under operating, then a layer of continuous water curtain will be formed by water overflowing the water tank. After that, water flow into the reservoir again to form an open cycle.

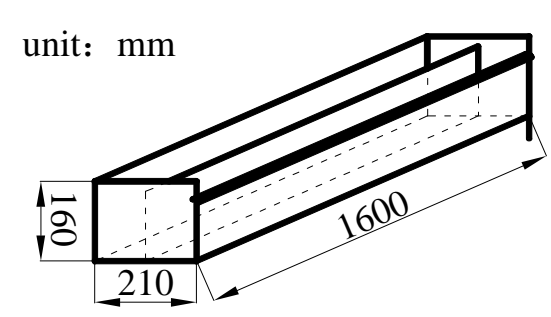

Fig. 10 Box structure.

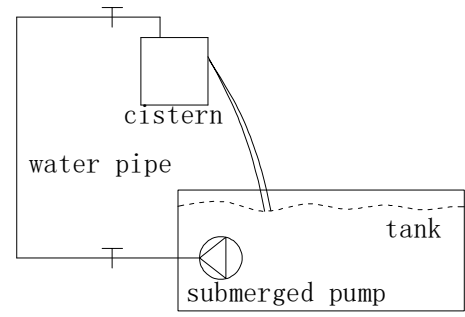

Fig. 11 Experimental device works.

Experiment includes: 1) The formation of continuous water curtain through half arc outlet; 2) As in practical application, the wider the continuous water curtain is, the better smoke control results will be get, so, the change of formation of continuous water curtain will be observed by changing the surface tension of the water, which can be changed by adding a certain amount of detergent into the water.

Table 1. Test results of different conditions.

\begin{tabular}{cccc}
\hline Conditions & $\begin{array}{c}\text { Continuous water curtain water } \\
\text { overflow length }(\mathrm{mm})\end{array}$ & $\begin{array}{c}\text { Continuous water curtain width of the } \\
\text { water at the bottom of the overflow }(\mathrm{mm})\end{array}$ & $\begin{array}{c}\text { Washing dose } \\
\left(\mathrm{L} / \mathrm{m}^{3}\right)\end{array}$ \\
\hline 1 & 1100 & 800 & 0 \\
2 & 1050 & 970 & 0.196 \\
3 & 1030 & 950 & 0.297 \\
4 & 1000 & 950 & 0.403 \\
5 & 1020 & 950 & 0.522 \\
7 & 1050 & 900 & 0.660 \\
8 & 1050 & 950 & 0.811 \\
\hline
\end{tabular}

Working Condition Test. There are 8 test conditions in total. Condition 1: Testing without adding detergent; Condition 2 8: Testing under different amount of detergent (using as reduce the surface 
tension of the water [7]), the total water flow remains steady during testing. Table 1 below shows the test results, Fig. 12 shows the experiment.

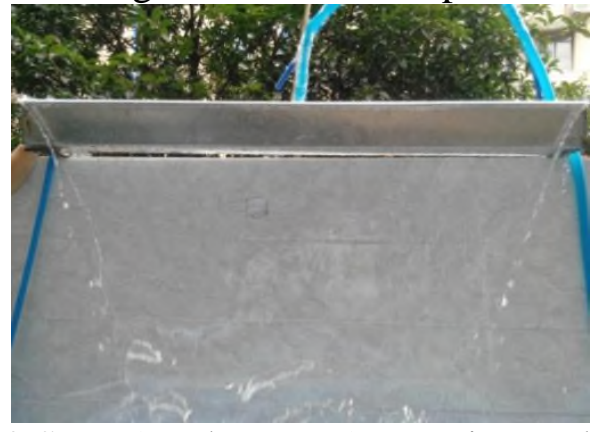

Fig. 12 State (no detergent) experimental water curtain formed.

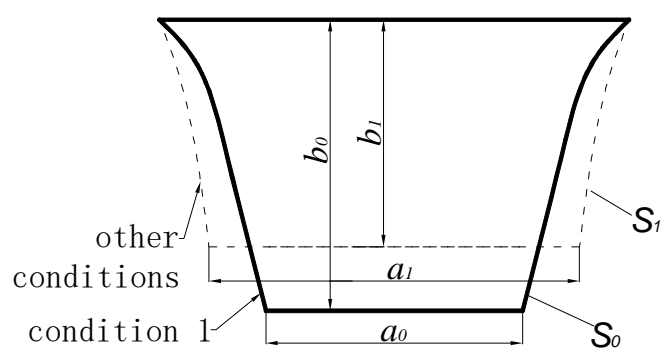

Fig. 13 Water curtain shape change contrast.

Result Analyze. Condition 1 in Table 1 and Fig. 12 show that the water curtain in the experiment shape into inverted trapezoidal, and the horizontal width of water curtain gradually shrinks along the down-flow direction. When the overflow reaches a certain length, the continuous water curtain will suddenly scattered because of the air involvement, then the water become discontinuous. In Fig. 5, Fig. 8 and Fig. 12 compared analysis, the trajectory of water curtain boundary is the shape of the curve, but continuous water curtain shape approximation is in the shape of inverted.

In condition 2 8, the length and width of the overflow change with the surface tension of the water, which testify that surface tension of water can influence the shape of water curtain. But the length and width of overflow act out in reciprocal trend (Fig. 13), more relevant parameters about shape changing needed to be found. The parameters to be discussed next are area change rate, width change rate, length change rate and width change rate converted to $1000 \mathrm{~mm}$ overflow length under different conditions.

From the experiment, the water curtain shape is close to the shape of inverted trapezoid, therefore, the continuous water curtain is calculated as trapezoid.

Calculation formula of water curtain area:

$$
S=\frac{1600+a}{2} \times b \times 10^{-6}
$$

Type: $S$ - continuous water curtain area, $\mathrm{m}^{2}$;

$a$-bottom width of overflow of continuous water curtain , $\mathrm{mm}$;

$b$ - continuous water curtain overflow length, $\mathrm{mm}$.

Calculation formula of area growth rate:

$\eta_{s}=\frac{S_{1}-S_{0}}{S_{0}} \times 100 \%$

Type: $\eta_{s}$-area growth rate, \%;

$S_{1}$ - continuous water curtain area under working condition $1, \mathrm{~m}^{2}$;

$S_{0}$-continuous water curtain area under other working conditions, $\mathrm{m}^{2}$.

Calculation formula of width growth rate:

$\eta_{a}=\frac{a_{1}-a_{0}}{a_{0}} \times 100 \%$

Type: $\eta_{a}$-width growth rate, $\%$;

$a_{0}$ - continuous water curtain width under working condition $1, \mathrm{~mm}$;

$a_{1}$-continuous water curtain width under other working conditions ,mm.

Calculation formula of length growth rate: 
$\eta_{b}=\frac{b_{1}-b_{0}}{b_{0}} \times 100 \%$

Type: $\quad \eta_{b}$-length growth rate, $\%$;

$b_{0}$-continuous water curtain length under working condition $1, \mathrm{~mm}$;

$b_{1}$ - continuous water curtain length under other working conditions, $\mathrm{mm}$.

Calculation formula by using triangle similarity theorem, converted the water curtain width to $1000 \mathrm{~mm}$ overflow length:

$$
a_{b=1000}=1600-\frac{1000}{b} \times(1600-a)
$$

Type: $a_{b=1000}$ - The water curtain width converted to $1000 \mathrm{~mm}$ overflow length, $\mathrm{mm}$.

Calculation formula of width growth rate:

$$
\eta_{a_{b=1000}}=\frac{a_{b=1000(\text { other working conditions) }}-a_{b=1000(\text { working condition } 1)}}{a_{b=1000(\text { working condition } 1)}}
$$

Type: $\eta_{a_{b=1000}}$ - width growth rate, $\%$;

$a_{b=1000 \text { (working condition 1) }}$ - water curtain width when the continuous water curtain length is $1000 \mathrm{~mm}$ under working condition $1, \mathrm{~mm}$;

$a_{b=1000 \text { (other working conditions) }}$-water curtain width when the continuous water curtain length is $1000 \mathrm{~mm}$ under other working conditions, $\mathrm{mm}$

Table 2 shows the experiment data.

Table 2. Changeable parameter analysis.

\begin{tabular}{ccccccc}
\hline Conditions & $S\left(\mathrm{~m}^{2}\right)$ & $(\%)$ & $(\%)$ & $(\%)$ & $(\mathrm{mm})$ & $(\%)$ \\
\hline 1 & 1.32 & $/$ & $/$ & $/$ & 872.73 & $/$ \\
2 & 1.35 & 2.22 & 21.25 & -4.55 & 1000.00 & 14.59 \\
3 & 1.31 & -0.51 & 18.75 & -6.36 & 968.93 & 11.03 \\
4 & 1.28 & -3.41 & 18.75 & -9.09 & 950.00 & 8.86 \\
5 & 1.30 & -1.48 & 18.75 & -7.27 & 962.75 & 10.32 \\
6 & 1.31 & -0.57 & 12.50 & -4.55 & 933.33 & 6.95 \\
7 & 1.34 & 1.42 & 18.75 & -4.55 & 980.95 & 12.40 \\
8 & 1.38 & 4.17 & 12.50 & 0.00 & 963.64 & 10.42 \\
average & $/$ & 0.26 & 17.32 & -5.19 & 965.66 & 10.65 \\
\hline
\end{tabular}

Table 2 and Fig. 14 show that surface tension of water reduced as the amount of detergent increased, the area change rate of water curtain first decreased then increased, but the change is small, with the average change rate is $0.26 \%$. The change of water surface tension has little influence on the whole water curtain area; Minimum width change rate shows a trend of weak reducing, with the average change rate is $17.32 \%$, after conversion to the condition of unified $1000 \mathrm{~mm}$ length overflow, the average width change rate is $10.65 \%$. The width of water curtain increases as the water surface tension decreases; The length change rate of water curtain first decreased then increased, the data is negative, with the average change rate is $-5.19 \%$, which shows the length of the water curtain decreases as the water surface tension decreases. 


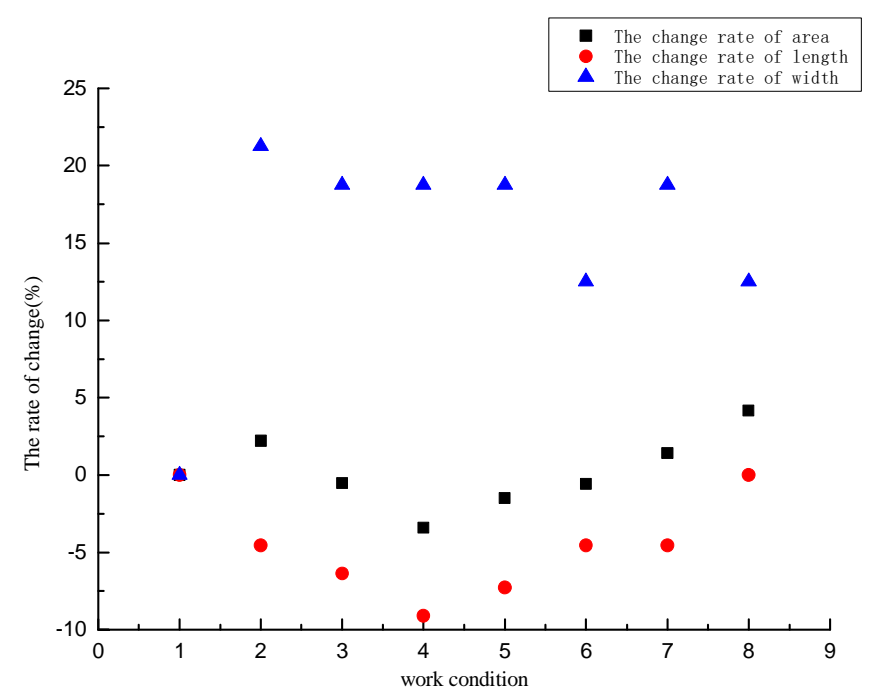

Fig. 14 Parameters change trend under different detergent conditions.

\section{Conclusion}

Through theoretical analysis, numerical simulation and experimental research, come out the following conclusions:

1) Continuous water curtain boundary surface is influenced by the resultant action from air friction $(f)$, atmospheric pressure $(\mathrm{P})$, water surface tension $(\mathrm{F})$ and gravity $(\mathrm{G})$, which result in the shrinkage at the down-flow stage. The fluid trajectory on boundary surface shapes in curve. But continuous water curtain shape approximation is in the shape of inverted.

2) Through theoretical analysis and simulation verification, flow velocity on the boundary surface of continuous water curtain is lower than that on the non-boundary surface;

3) To decrease the water surface tension, the influence on water curtain area change is small, but the width will increase and the length will decrease.

\section{Reference}

[1] Y. Yu, Y. Chu, D. Liang, Study on Smoke Control Strategy in a High-rise Building Fire, Procedia Eng. 71 (2014) 145-152.

[2] L. R. Zhang, B. H. Pang, Landscape waterfalls numerical simulation based on the theory of fluid dynamics, Water supply and drainage, 12 (2014) 120-123.

[3] G. Q. Liu, fine particle discrete dynamics research under van der Waals force and static electricity power, Tsinghua university, 2011.

[4] J. Zhang, L. Yang, etc. Study on Mechanism of liquid Surface Tension and Analysis of Direction, China Pet. Chem. Ind. Stand. Qual. 5 (2012) 73-74.

[5] D. F. Parsons, R. B. Walsh, V. S. J. Craig, Surface forces: Surface roughness in theory and experiment, J. Chem. Phys. 140(16470116) (2014) 164701.

[6] C. Shi, X. Cui, L. Xie, et al. Measuring Forces and Spatiotemporal Evolution of Thin Water Films between an Air Bubble and Solid Surfaces of Different Hydrophobicity, Acs. Nano. 9(1) (2015) 95-104.

[7] K. Yokoi, M. Kawaguchi, Effect of temperature on surface pressure-area isotherms and surface dilational moduli of poly ( $\mathrm{N}$-isopropyl acrylamide) monolayers spread at air-water interface, Colloids and Surfaces A: Physicochem. Eng. Aspects, 457 (2014) 469-475.

[8] J. Cheng, L. Li, K. Liu, Experimental research on the relationship between liquid surface tension coefficient and concentration, Chin. test, 40(3) (2014) 32-34. 